\title{
Effect of a Modest Weight Loss in Normalizing Blood Pressure in Obese Subjects on Antihypertensive Drugs
}

\author{
Luisa Gilardini Gabriella Redaelli Marina Croci Antonio Conti \\ Lucia Pasqualinotto Cecilia Invitti
}

Department of Medical Sciences and Rehabilitation, IRCCS, Istituto Auxologico Italiano, Milan, Italy

\author{
Key Words \\ Obesity · Hypertension · Lifestyle intervention · Weight loss
}

\begin{abstract}
Objective: To assess the effect of a lifestyle intervention in lowering/normalizing blood pressure (BP) levels in hypertensive (controlled or not) obese patients. Methods: In this prospective observational study, 490 obese hypertensive patients, 389 controlled (BP < 140/90 mm $\mathrm{Hg} ; \mathrm{CH}$ ) and 101 uncontrolled (BP $\geq 140 / 90 \mathrm{~mm} \mathrm{Hg} ; \mathrm{UH}$ ) attended a 3-month lifestyle intervention. Before and after the intervention we assessed weight, waist circumference, fat mass, $\mathrm{BP}$, metabolic and renal variables, and physical activity. A multivariate regression model was used to determine the predictors of BP changes. Results: $18.9 \%$ of $\mathrm{CH}$ and $20.0 \%$ of UH were on $\geq 3$ antihypertensive drugs. Weight change (average $-4.9 \pm 2.7 \%$ ) was independent of the antihypertensive drugs employed. Systolic BP (SBP) decreased by $23 \mathrm{~mm} \mathrm{Hg}$ and diastolic BP (DBP) by $9 \mathrm{~mm} \mathrm{Hg}$, in patients with UH most of whom (89\%) normalized BP levels (in 49\% after a weight loss $<5 \%$ ). Age, gender, whole and central obesity, concomitance of type 2 diabetes, chronic renal disease, physical activity intensification, and pharmacological therapy did not affect BP lowering. In the regression analysis with SBP change as dependent variable, weight reduction ( $\beta=0.523, p=0.005)$ and group (UH vs. $C H, \beta=-19.40, p=0.0005)$ remained associated with SBP reduction. When DBP change was entered as dependent variable, baseline uric acid remained associated with DBP reduction $(\beta=0.824, p<0.05)$. Conclusion: Lifestyle interventions are useful for all obese hypertensive patients in most of whom a modest weight loss is sufficient to normalize BP levels avoiding the aggressive use of multiple antihypertensive drugs.


Gilardini et al.: Effect of a Modest Weight Loss in Normalizing Blood Pressure in Obese Subjects on Antihypertensive Drugs

\section{Introduction}

Raised BMI is a major risk factor for cardiovascular diseases, which are the leading cause of death. Hypertension is very common in obese patients and is linked to major risk factors for premature death, such as hyperglycemia and physical inactivity [1]. Obesity-related hypertension is a distinct phenotype in view of its complex pathophysiology that involves adipose tissue dysfunction, adipokine alterations, insulin resistance, dysfunctional immunity, inappropriate activity of the sympathetic nervous and renin-angiotensin-aldosterone systems, and abnormal renal and vascular function [2]. Management of obesity-related hypertension is a major challenge. The pathophysiological key of this form of hypertension is excessive fat accumulation, thus weight reduction is recommended as the first-line therapy for the treatment of obesity-related hypertension [3]. Despite the overwhelming evidence that weight reduction lowers blood pressure (BP) in hypertensive and non-hypertensive obese patients, weight loss does not lower BP in all persons [3-5], and some issues remain unclear. For instance, whether or not weight loss is effective in reducing BP in obese patients under combined antihypertensive therapy is unknown. Furthermore, although the weight loss required to reduce BP is reported to be higher than that required to decrease triglycerides and glucose [6], the amount of weight loss needed to normalize BP in obese hypertensive individuals is undefined. The AHA/ACC/TOS Guideline for the management of overweight and obesity in adults reported that in obese subjects with elevated cardiovascular risk, systolic BP (SBP) and diastolic BP (DBP) decreased by approximately 3 and $2 \mathrm{~mm} \mathrm{Hg}$, respectively, after a $\geq 5 \%$ weight loss, with modest and variable reductions in $\mathrm{BP}$ at $<5 \%$ weight loss [6]. Lastly, it is unclear whether or not the concomitance of cardiometabolic risk factors affects the BP-lowering efficacy of weight loss [7, 8].

We conducted a prospective observational study in a large cohort of obese patients under one or more antihypertensive drugs to assess: i) the effect of a 3-month lifestyle intervention in lowering BP, ii) the extent of weight loss needed to normalize BP levels, iii) whether the concomitance of cardiometabolic risk factors (type 2 diabetes, dyslipidemia, chronic renal disease), BP control, and antihypertensive therapy affect the BP-lowering effects of lifestyle intervention.

\section{Patients and Methods}

The study sample consisted of 490 Caucasian obese hypertensive patients. Subjects were recruited between 2008 and 2014 from those patients referred to the IRCCS, Istituto Auxologico Italiano for a weight loss lifestyle intervention. Prerequisite for entering the study was being under one or more antihypertensive drugs for at least 6 months with good treatment compliance. Patients with secondary and drug-induced hypertension were excluded. Information on smoking habits, use of medication, and family history of obesity, diabetes and cardiovascular disease was collected from all subjects.

Participants entered a 3-month lifestyle intervention which consisted of weekly individual sessions for nutritional education, peer group psychological support and physical activity in the gym with a trainer who provided advices on exercise activity. A self-monitor diary, including food consumption, daily physical activity and emotional reactions, was used as a tool for education and reinforcement. Daily caloric requirement was calculated using the Harris-Benedict equation and an individual activity factor. A diet based on a $500-\mathrm{kcal} /$ day deficit from the individual estimated caloric requirement was prescribed. The diet which was high in vegetables and low in salt and simple sugars consisted of $25 \%$ of total energy intake as protein, $20 \%$ as fat and $55 \%$ as carbohydrate. Fresh foods, at least three fish meals per week, and avoiding alcohol were recommended. The prescribed physical activity program consisting of $70 \%$ moderate-intensity aerobic physical activity and 30\% muscle-strengthening activities lasted $210 \mathrm{~min} /$ week. The patient's compliance to diet and exercise was recorded at each session. 
Gilardini et al.: Effect of a Modest Weight Loss in Normalizing Blood Pressure in Obese Subjects on Antihypertensive Drugs

Before and after the 3-month lifestyle intervention, anthropometric measures, BP, and heart rate were measured, and body composition assessed using bioelectric impedance assay (BIA 101-RJL Systems Akern srl, Firenze, Italy). Heart rate was used as a proxy of cardiovascular fitness. Waist circumference was measured at the level of the umbilicus. Trained certified individuals measured BP using a standard mercury sphygmomanometer and a cuff size optimized for arm circumference. Three BP measurements separated by 5 min were obtained in a sitting position. SBP and DBP were identified using phase I and V (disappearance) Korotkoff sounds. Patients were defined as having uncontrolled hypertension if their mean BP levels $\geq$ $140 / 90 \mathrm{~mm} \mathrm{Hg}$, despite pharmacological treatment. A blood sample was taken for the measurement of glucose, high-density lipoprotein (HDL) and low-density lipoprotein (LDL) cholesterol, triglycerides, uric acid, and creatinine. Three morning urine samples were collected for the measurement of the albumin/creatinine ratio. Microalbuminuria was defined by mean albumin/creatinine ratio $\geq 2.5$ and $3.5 \mathrm{mg} / \mathrm{mmol}$ for men and women, respectively.

In non-diabetic subjects, glucose and insulin were measured after the oral glucose tolerance test. Impaired fasting glucose (IFG) and impaired glucose tolerance (IGT) were defined using the American Diabetes Association criteria [9]. Dyslipidemia was defined by the presence of high triglycerides $(\geq 150$ $\mathrm{mg} / \mathrm{dl}$ ) and/or low HDL cholesterol ( $<40 \mathrm{mg} / \mathrm{dl}$ in men and $<50 \mathrm{mg} / \mathrm{dl}$ in women) or the use of lipid-lowering therapy. Glomerular filtration rate (GFR) was estimated using the recalibrated version of the four-variable Modification of Diet in Renal Disease study equation [10].

Before and after lifestyle intervention, physical activity was evaluated with the International Physical Activity Questionnaire (IPAQ) - short version that compute the metabolic equivalent (MET = time spent in physical activity, expressed in minutes per week) [11]. Number of days and minutes in the last week spent in vigorous activity, moderate activity and walking were assessed.

The Ethics Committee of our Institute approved the study, and all subjects gave their informed consent after we provided a full explanation of the study.

\section{Biochemical Measurements}

Glucose, lipids, uric acid, and creatinine were measured using an automated analyzer (Roche Diagnostics, Mannheim, Germany). Insulin was measured by a chemiluminescent assay (Roche Diagnostics) with a sensitivity of $0.2 \mu \mathrm{U} / \mathrm{ml}$ as well as an intra- and inter-assay CV of 3.3 and $4.1 \%$, respectively. Urine albumin excretion was determined by an immunoturbidimetric assay (Roche Diagnostics).

\section{Statistical Analyses}

Variables that were not normally distributed were log transformed for the analysis. Changes induced by lifestyle intervention were calculated using the following ratio: ((value at month 3 - baseline value) / baseline value) $\times 100$. Continuous variables were expressed as means \pm SD or median (interquartile range) for variables that were not normally distributed and categorical data as proportions. T-test and chi square test were used when appropriate to test differences in clinical characteristics between patients with uncontrolled and controlled hypertension at baseline. Univariate regression models were fitted with continuous and categorical variables to estimate the association between each clinical variable and changes in BP. All variables with $\mathrm{p}<0.20$ were eligible to be included in a multivariate regression model to determine predictors of changes in BP. For all included variables, the interaction with the variable group was examined. All analyses were performed using SAS version 9.4 software (SAS Institute, Cary, NC, USA). For all statistical analyses, significance was set at $\mathrm{p} \leq 0.05$ except for interaction term in multivariate regression model where significance was set at $\mathrm{p}<0.20$ [12].

The sum of MET (min/week) of moderate activity, vigorous activity and walking was used to assess total physical activity of subjects.

\section{Results}

\section{Characteristics of Obese Hypertensive Patients}

Clinical characteristics of patients are shown in table 1 . About $20 \%$ subjects in both groups were on $\geq 3$ antihypertensive drugs. The antihypertensive drugs taken by patients were similar in the 389 hypertensive patients with $\mathrm{BP}<140 / 90 \mathrm{~mm} \mathrm{Hg}$ (controlled hyper- 
Gilardini et al.: Effect of a Modest Weight Loss in Normalizing Blood Pressure in Obese Subjects on Antihypertensive Drugs

Table 1. Baseline characteristics of 389 obese patients with controlled hypertension and 101 with uncontrolled hypertension $^{\mathrm{a}}$

\begin{tabular}{|c|c|c|}
\hline & $\begin{array}{l}\text { Controlled hypertension } \\
(\mathrm{n}=389)\end{array}$ & $\begin{array}{l}\text { Uncontrolled hypertension } \\
(\mathrm{n}=101)\end{array}$ \\
\hline Age, years & $58.9 \pm 10.1$ & $60.6 \pm 10.3$ \\
\hline Men, \% & 26.1 & 28.0 \\
\hline FH of obesity, \% & 60.9 & 68.7 \\
\hline FH of diabetes, $\%$ & 54.2 & 44.4 \\
\hline $\mathrm{FH}$ of $\mathrm{CVD}, \%$ & 49.2 & 41.8 \\
\hline $\mathrm{BMI}, \mathrm{kg} / \mathrm{m}^{2}$ & $37.1 \pm 4.5$ & $39.1 \pm 5.3^{* *}$ \\
\hline Weight, kg & $96.7 \pm 15.8$ & $101.9 \pm 17.9^{*}$ \\
\hline Waist circumference, $\mathrm{cm}$ & $117.1 \pm 10.4$ & $120.9 \pm 11.6^{*}$ \\
\hline Fat mass, $\%$ & $46.3 \pm 6.4$ & $46.5 \pm 7.5$ \\
\hline Fat free mass, $\%$ & $53.7 \pm 6.8$ & $53.5 \pm 7.5$ \\
\hline $\mathrm{SBP}, \mathrm{mm} \mathrm{Hg}$ & $127.4 \pm 9.2$ & $153.3 \pm 9.8^{* *}$ \\
\hline $\mathrm{DBP}, \mathrm{mm} \mathrm{Hg}$ & $78.9 \pm 5.7$ & $87.5 \pm 8.8^{* *}$ \\
\hline Heart rate, beats/min & $74.7 \pm 12.8$ & $73.3 \pm 12.3$ \\
\hline Fasting glucose, $\mathrm{mg} / \mathrm{dl}$ & $104.3 \pm 29.7$ & $109.9 \pm 41.3$ \\
\hline Fasting insulin, $\mu \mathrm{U} / \mathrm{ml}$ & $11.5(7.8-18.5)$ & $13.0(9.5-18.9)$ \\
\hline HDL cholesterol, mg/dl & $53.3 \pm 15.1$ & $54.1 \pm 12.1$ \\
\hline LDL cholesterol, mg/dl & $123.0 \pm 33.2$ & $127.9 \pm 62.9$ \\
\hline Triglycerides, mg/dl & $120(91-149)$ & $117(83-140)$ \\
\hline Uric acid, mg/dl & $5.8 \pm 1.3$ & $5.9 \pm 1.2$ \\
\hline AER mg/mmol & $0.6(0.3-0.1)$ & $0.5(0.3-1.4)$ \\
\hline $\mathrm{GFR}, \mathrm{ml} / \mathrm{min}$ & $76.2 \pm 16.6$ & $77.5 \pm 20.0$ \\
\hline Physical activity (MET), min/week & $495(240-1,040)$ & $280(181-815)$ \\
\hline$\geq 3$ anti-hypertensive drugs, $\%$ & 18.9 & 20.0 \\
\hline Angiotensin-converting enzyme inhibitors, \% & 33.7 & 32.0 \\
\hline Angiotensin receptor blockers, $\%$ & 41.9 & 41.0 \\
\hline Calcium antagonists, $\%$ & 18.5 & 16.0 \\
\hline Diuretics, $\%$ & 44.0 & 53.0 \\
\hline$\beta$-blockers, \% & 35.2 & 39.0 \\
\hline$\alpha$-blockers, $\%$ & 4.1 & 7.0 \\
\hline Tobacco use, $\%$ & 8.9 & 8.1 \\
\hline Diabetes/IGT/IFG, \% & $22.1 / 14.4 / 24.2$ & 21.4/14.1/32.2 \\
\hline Dyslipidemia, $\%$ & 55.8 & 43.9 \\
\hline Microalbuminuria, \% & 15.9 & 17.3 \\
\hline GFR $<60 \mathrm{ml} / \mathrm{min}, \%$ & 14.3 & 15.2 \\
\hline
\end{tabular}

$\mathrm{AER}=$ albumin excretion rate; $\mathrm{CVD}=$ cardiovascular disease; $\mathrm{FH}$ = family history

${ }^{*} \mathrm{p}<0.005,{ }^{* *} \mathrm{p}<0.0001$.

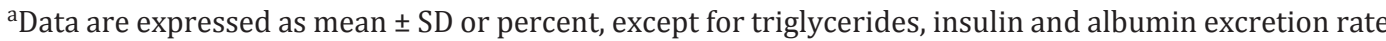
that are expressed as median (interquartile range).

tension) and in the 101 patients with $\mathrm{BP} \geq 140 / 90 \mathrm{~mm} \mathrm{Hg}$ (uncontrolled hypertension). Doses of antihypertensive drugs was kept unchanged throughout the study.

Patients with uncontrolled hypertension were more obese, but had similar age, sex, tobacco use, family history of obesity, diabetes, and cardiovascular diseases as well as similar proportions of microalbuminuria, chronic renal disease (GFR $<60 \mathrm{ml} / \mathrm{min}$ ), type 2 diabetes, prediabetes, and dyslipidemia than those with controlled hypertension.

\section{Effects of 3-Month Lifestyle Intervention}

All subjects completed the 3-month lifestyle intervention. In the whole group of 490 subjects there was a significant reduction in anthropometric measures ( $\Delta$ weight $-4.9 \pm 2.7 \%$, 
Gilardini et al.: Effect of a Modest Weight Loss in Normalizing Blood Pressure in Obese Subjects on Antihypertensive Drugs

Table 2. Multivariate regression analysis for variables associated with SBP (panel A) and DBP change (panel B)

\begin{tabular}{|c|c|c|c|c|c|}
\hline \multicolumn{3}{|l|}{ Panel A } & \multicolumn{3}{|l|}{ Panel B } \\
\hline & $\beta$ & $\mathrm{p}$ & & $\beta$ & $\mathrm{p}$ \\
\hline $\begin{array}{l}\text { Group* uncontrolled vs. } \\
\text { controlled hypertension }\end{array}$ & -19.400 & 0.0004 & $\begin{array}{l}\text { Group* uncontrolled vs. } \\
\text { controlled hypertension }\end{array}$ & -1.450 & 0.8350 \\
\hline HDL cholesterol & -0.068 & 0.0634 & Family history of CVD & 0.501 & 0.6285 \\
\hline Group* HDL cholesterol & 0.102 & 0.2324 & Group* family history of CVD & 2.103 & 0.3590 \\
\hline Weight change & 0.523 & 0.0047 & LDL cholesterol & -0.015 & 0.4681 \\
\hline Group* weight change & -0.591 & 0.1108 & Group* LDL cholesterol & -0.014 & 0.6612 \\
\hline Triglycerides change & 0.020 & 0.2190 & Uric acid & 0.824 & 0.0408 \\
\hline Group* triglycerides change & 0.026 & 0.5306 & Group* uric acid & 0.708 & 0.4404 \\
\hline
\end{tabular}

CVD = Cardiovascular disease

Fig. 1. Relationship between the intervention-induced SBP decrease and weight loss in patients with uncontrolled (dashed line) and controlled (solid line) hypertension.

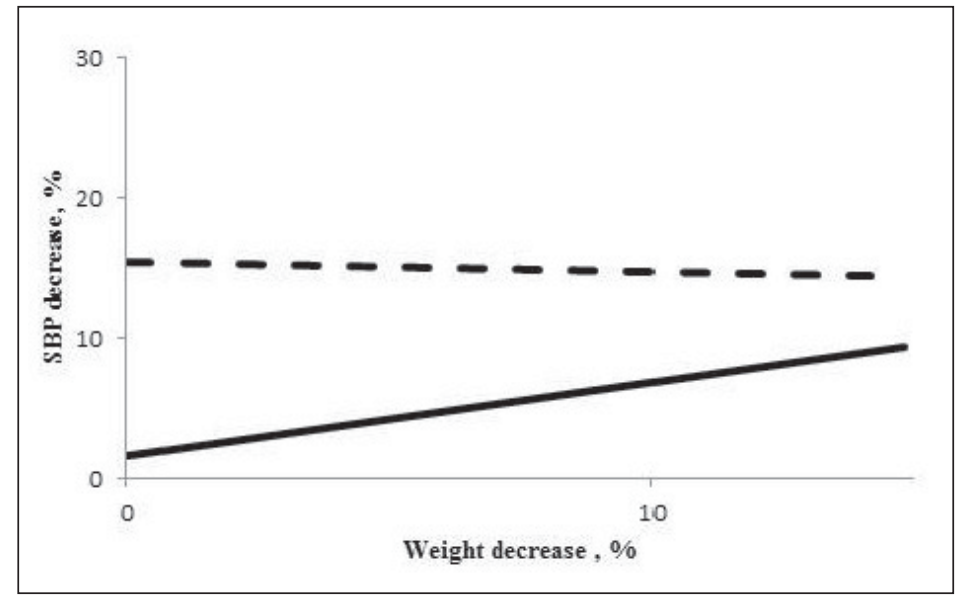

$\Delta$ waist circumference $-3.4 \pm 2.9 \%$; $<<0.0001)$, body fat $(-3.5 \pm 7.5 \% ; \mathrm{p}<0.0001), \mathrm{BP}(\Delta \mathrm{SBP}$ $-6.3 \pm 5.2 \%, \Delta$ DBP $-4.3 \pm 5.3 \% ; p<0.0001)$, heart rate $(-8.7 \pm 11.2 \% ; p<0.0001)$, and metabolic variables $(\Delta$ glucose $-3.1 \pm 11.3 \%, \Delta$ LDL cholesterol $-2.1 \pm 11.6 \%, \Delta$ uric acid $-4.6 \pm$ $16.4 \%$; $<0.0001)$ as well as a significant increase in physical activity $(\Delta$ MET $389 \pm 755 \%$; $\mathrm{p}<0.0001)$ and fat-free mass $(3.4 \pm 6.2 \% ; \mathrm{p}<0.0001)$. Weight change was independent of the antihypertensive drugs assumed.

SBP and DBP decreased by $23 \mathrm{~mm} \mathrm{Hg}$ and $9 \mathrm{~mm} \mathrm{Hg}$, respectively in patients with uncontrolled hypertension. BP levels normalized in $88.8 \%$ of these patients, $85 \%$ of whom was treated with $\geq 3$ antihypertensive drugs. BP normalization was obtained in $49 \%$ of uncontrolled patients after a $<5 \%$ weight loss.

\section{Predictors of BP Changes}

Entering in the univariate analysis all variables listed in table 1 and their changes after the intervention, the SBP change was correlated with the variable group (uncontrolled vs. controlled hypertension, $\beta=-10.9, p<0.0001)$, baseline HDL cholesterol $(\beta=-0.04, p=$ $0.130)$, HOMA-IR levels $(\beta=0.50, p<0.001)$, weight change $(\beta=0.49, p<0.005)$, and triglyceride change $(\beta=0.02, p=0.120)$.

DBP change was correlated with group $(\beta=-6.72, p<0.0001)$, heart rate $(\beta=-0.06, p=$ $0.120)$, LDL cholesterol $(\beta=-0.02, p=0.150)$, uric acid $(\beta=0.56, p=0.120)$, family history of 
Gilardini et al.: Effect of a Modest Weight Loss in Normalizing Blood Pressure in Obese Subjects on Antihypertensive Drugs

cardiovascular diseases $(\beta=1.28, p=0.160)$, and changes in heart rate $(\beta=0.06, p=0.140)$ and albumin excretion rate $(\beta=0.005, p=0.080)$.

In the multivariate regression analysis (table 2, panel A), weight reduction, group (uncontrolled vs. controlled hypertension), and interaction term remained associated with SBP reduction. In other words, the relationship between the intervention-induced SBP decrement and weight loss tended to be greater in patients with uncontrolled than in those with controlled hypertension (fig. 1).

Baseline uric acid remained associated with DBP reduction (table 2. panel B). This indicates that intervention-induced decrement of SBP was higher in uncontrolled than in controlled hypertensive patients, and DBP reduction was lower in subjects with higher uric acid levels.

\section{Discussion}

Results from this study indicate that BP levels normalize with lifestyle changes and a modest weight loss within a short time in most obese subjects independently of age, sex, degree of total and central obesity, and type and number of antihypertensive drugs consumed.

In particular, lifestyle intervention decreases SBP by $23 \mathrm{~mm} \mathrm{Hg}$ and DBP by $9 \mathrm{~mm} \mathrm{Hg}$ in subjects with uncontrolled hypertension with most of whom were on $\geq 3$ antihypertensive drugs. The magnitude of this BP reduction is comparable to that obtained after adding another antihypertensive drug to the treatment regimen, but with the additional benefit of improving other cardiovascular risk factors such as glucose, LDL and HDL cholesterol, and uric acid levels.

The AHA/ACC/TOS Guideline for the management of overweight and obesity in adults highlighted that weight loss of as little as 3-5\%, despite reducing triglycerides and glucose levels, is insufficient to decrease BP levels [6]. Our data demonstrate that even a weight loss $<5 \%$ obtained with diet and physical activity may normalize BP levels. Few studies investigated the effect of weight loss in uncontrolled hypertension [13], and most excluded patients with BP values $>140 / 90$ [13] or $>160 / 100 \mathrm{~mm} \mathrm{Hg}$ [7].

Studies in obese hypertensive patients reported that the BP-lowering effect of lifestyle intervention is independent of baseline obesity $[14,15]$. In the present study, we observed that also central obesity and fat mass do not affect the benefits of weight loss.

Several studies in obese hypertensive subjects found a strong correlation between changes in BP and weight induced by different weight-reducing diets $[7,15,16]$. We here observed that weight changes are associated with SBP changes, but this relation is ascribable only to patients with controlled hypertension. This finding confirms the relevant role of fat mass reduction in lowering BP through the improvement of adipocyte and sympathetic dysfunction, and reduction of local activity of the adipose renin-angiotensin-aldosterone system [2]. However, our results also suggest that in patients with uncontrolled hypertension under pharmacological therapy the reduction in body fatness is not the major determinant of $\mathrm{BP}$ reduction. It can be assumed that dietary changes such as the reduction in salt and fructose intake and the increase in vegetable protein are largely responsible for the BP decrease in these subjects [17].

The present study does not allow identifying whether diet or physical activity had a greater weight in lowering BP. However, the lack of association between changes in BP and physical activity suggests that in our cohort of obese hypertensive subjects physical activity played a minor role. Accordingly, it has been reported that body fat reduction obtained with aerobic exercises without dietary intervention is often ineffective in lowering BP $[18,19]$ and that adding regular physical to antihypertensive drugs has no additive effects on BP [20]. 
Gilardini et al.: Effect of a Modest Weight Loss in Normalizing Blood Pressure in Obese Subjects on Antihypertensive Drugs

Furthermore, in our cohort the concomitance of cardiometabolic alterations (type 2 diabetes, dyslipidemia, and chronic renal disease) did not affect the weight loss-induced BP decrease.

We observed that DBP levels decreased to a lower extent in subjects with higher levels of uric acid. This finding supports the concept that hyperuricemia and obesity have an additive role on the risk of hypertension and might induce hypertension by specific mechanisms such as the increase in $\mathrm{Na}^{+}$reabsorption [21,22].

The major limitation of this study is the lack of a control group. However, our study was not aimed at comparing the lowering BP of lifestyle intervention with that of the usual therapeutic care, but rather to establish if lifestyle intervention reduces BP independently of clinical conditions and of what weight loss is needed to obtain BP normalization. Moreover, the study does not permit to determine whether the duration of hypertension influences the response to lifestyle interventions.

In conclusion, this study provides the evidence that lifestyle interventions are useful for all obese hypertensive patients, and in most subjects a modest weight loss is sufficient to normalize BP levels, avoiding the aggressive use of multiple antihypertensive drugs.

\section{Acknowledgements}

We thank Prof. Antonella Zambon and Dr. Davide Soranna for statistical analysis.

\section{Disclosure Statement}

The authors declare no conflict of interest.

\section{References}

1 World Health Organization. Obesity and overweight. Fact sheet N311.Updated March 2011;www.who.int/ mediacentre/factsheets/fs311/en/index.html (last accessed July 14, 2016).

2 DeMarco VG, Aroor AR, Sowers JR: The pathophysiology of hypertension in patients with obesity. Nat Rev Endocrinol 2014;10:364-376.

3 Mancia G, Fagard R, Narkiewicz K, Redon J, Zanchetti A, Böhm M, et al: 2013 ESH/ESC practice guidelines for the management of arterial hypertension. Task Force for the Management of Arterial Hypertension of the European Society of Hypertension and the European Society of Cardiology. Blood Press 2014;23:3-16.

4 Hallersund P, Sjöström L, Olbers T, Lönroth H, Jacobson P, Wallenius V, et al: Gastric bypass surgery is followed by lowered blood pressure and increased diuresis - long term results from the Swedish Obese Subjects (SOS) study. PLoS One 2012; 7:e49696.

5 Masuo K, Mikami H, Ogihara T, Tuck ML: Differences in mechanisms between weight loss-sensitive and -resistant blood pressure reduction in obese subjects. Hypertens Res 2001;24:371-376.

6 Jensen MD, Ryan DH, Apovian CM, Ard JD, Comuzzie AG, Donato KA, et al; American College of Cardiology/ American Heart Association Task Force on Practice Guidelines; Obesity Society: 2013 AHA/ACC/TOS guideline for the management of overweight and obesity in adults: a report of the American College of Cardiology/ American Heart Association Task Force on Practice Guidelines and The Obesity Society. Circulation;129(25 suppl 2):S102-138.

7 Wing RR, Lang W, Wadden TA, Safford M, Knowler WC, Bertoni AG, et al; Look AHEAD Research Group: Benefits of modest weight loss in improving cardiovascular risk factors in overweight and obese individuals with type 2 diabetes. Diabetes Care 2011;34:1481-1486.

8 Howden EJ, Leano R, Petchey W, Coombes JS, Isbel NM, Marwick TH: Effects of exercise and lifestyle intervention on cardiovascular function in CKD. Clin J Am Soc Nephrol 2013;8:1494-1501.

9 American Diabetes Association: Diagnosis and classification of diabetes mellitus. Diabetes Care 2013;36(suppl 1):S67-74.

10 Chronic Kidney Disease Epidemiology Collaboration: Expressing the Modification of Diet in Renal Disease Study equation for estimating glomerular filtration rate with standardized serum creatinine values. Clin Chem 2007;53:766-772. 
Gilardini et al.: Effect of a Modest Weight Loss in Normalizing Blood Pressure in Obese Subjects on Antihypertensive Drugs

11 Bauman AE, Booth ML, Ainsworth BE, et al: International Physical Activity Questionnaire: 12-country reliability and validity Med Sci Sports Exerc 2003;35:1381-1395.

12 Marshall SW: Power for tests of interaction: effect of raising the type I error rate. Epidemiol Perspect Innov 2007;4:1-7.

13 Calhoun DA, Jones D, Textor S, Goff DC, Murphy TP, Toto RD, et al; American Heart Association Professional Education Committee: Resistant hypertension: diagnosis, evaluation, and treatment: a scientific statement from the American Heart Association Professional Education Committee of the Council for High Blood Pressure Research. Circulation 2008;117:e510-526.

14 Goodpaster BH, Delany JP, Otto AD, Kuller L, Vockley J, South-Paul JE, et al: Effects of diet and physical activity interventions on weight loss and cardiometabolic risk factors in severely obese adults: a randomized trial. JAMA 2010;304:1795-1802.

15 Staessen J, Fagard R, Amery A: The relationship between body weight and blood pressure. J Hum Hypertens 1988;2:207-217.

16 Aucott L, Poobalan A, Smith WC, Avenell A, Jung R, Broom J: Effects of weight loss in overweight/obese individuals and long-term hypertension outcomes: a systematic review. Hypertension 2005;45:1035-1041.

17 Sacks FM, Svetkey LP, Vollmer WM, Appel LJ, Bray GA, Harsha D; et al; for the DASH-Sodium Collaborative Research Group: Effects on blood pressure of reduced dietary sodium and the dietary approaches to stop hypertension (DASH) diet. N Engl J Med 2001;344:3-10.

18 Trachta P, Drápalová J, Kaválková P, Toušková V, Cinkajzlová A, Lacinová Z, et al: Three months of regular aerobic exercise in patients with obesity improve systemic subclinical inflammation without major influence on blood pressure and endocrine production of subcutaneous fat. Physiol Res 2014;63(suppl 2):S299-308.

19 Montero D, Roche E, Martinez-Rodriguez A: The impact of aerobic exercise training on arterial stiffness in preand hypertensive subjects: a systematic review and meta-analysis. Int J Cardiol 2014;173:361-368.

20 Maruf FA, Akinpelu AO: Can aerobic exercise complement antihypertensive drugs to achieve blood pressure control in individuals with essential hypertension? J Cardiovasc Med 2014;15:456-462.

21 Han GM, Gonzalez S, DeVries D: Combined effect of hyperuricemia and overweight/obesity on the prevalence of hypertension among US adults: result from the National Health and Nutrition Examination Survey. J Hum Hypertens 2014;28:579-586.

22 Xu W, Huang Y, Li L, Sun Z, Shen Y, Xing J, et al: Hyperuricemia induces hypertension through activation of renal epithelial sodium channel (ENaC). Metabolism 2016;65:73-83. 